\title{
Fate of Dyspeptic or Colonic Symptoms After Laparoscopic Cholecystectomy
}

\author{
Gi Hyun Kim, ${ }^{1}$ Hyo Deok Lee, ${ }^{1}$ Min Kim,${ }^{1}$ Kyeongmin Kim, ${ }^{1}$ Yusook Jeong, ${ }^{1}$ Yong Joo Hong, ${ }^{1}$ Eun Seok Kang, ${ }^{1}$ Joung-Ho Han, ${ }^{1}$ \\ Jae-Woon $\mathrm{Choi}^{2}$ and Seon Mee Park ${ }^{1 *}$ \\ Departments of Internal Medicine and ${ }^{2}$ Surgery, Chungbuk National University College of Medicine, Cheongju, Chungcheongbuk-do, Korea
}

\begin{abstract}
Background/Aims
Gallbladder diseases can give rise to dyspeptic or colonic symptoms in addition to biliary pain. Although most biliary pain shows improvement after cholecystectomy, the fates of dyspeptic or colonic symptoms still remain controversial. This study assessed whether nonspecific gastrointestinal symptoms improved after laparoscopic cholecystectomy (LC) and identified the characteristics of patients who experienced continuing or exacerbated symptoms following surgery.
\end{abstract}

\section{Methods}

Sixty-five patients who underwent LC for uncomplicated gallbladder stones or gallbladder polyps were enrolled. The patients were surveyed on their dyspeptic or colonic symptoms before surgery and again at 3 and 6 months after surgery. Patients' mental sanity was also assessed using a psychological symptom score with the Symptom Checklist-90-Revised questionnaire.

Results

Forty-four (67.7\%) patients showed one or more dyspeptic or colonic symptoms before surgery. Among these, 31 (47.7\%) and $36(55.4 \%)$ patients showed improvement at 3 and 6 months after surgery, respectively. However, 18.5\% of patients showed continuing or exacerbated symptoms at 6 months after surgery. These patients did not differ with respect to gallstone or gallbladder polyps, but differed in frequency of gastritis. These patients reported lower postoperative satisfaction. Patients with abdominal symptoms showed higher psychological symptom scores than others. However, poor mental sanity was not related to the symptom exacerbation.

\section{Conclusions}

Elective LC improves dyspeptic or colonic symptoms. Approximately $19 \%$ of patients reported continuing or exacerbated symptoms following LC. Detailed history-taking regarding gastritis before surgery can be helpful in predicting patients' outcome after LC.

\section{(J Neurogastroenterol Motil 2014;20:253-260)}

\section{Key Words}

Brief Psychiatric Rating Scale; Cholecystectomy, laparoscopic; Gastritis; Postcholecystectomy syndrome

Received: October 7, 2013 Revised: December 18, 2013 Accepted: December 19, 2013

(c) This is an Open Access article distributed under the terms of the Creative Commons Attribution Non-Commercial License (http://creativecommons. org/licenses/by-nc/3.0) which permits unrestricted non-commercial use, distribution, and reproduction in any medium, provided the original work is properly cited.

*Correspondence: Seon Mee Park, MD, PhD

Department of Internal Medicine, Chungbuk National University College of Medicine, 52 Naesudong-ro, Heungdeok-gu, Cheongju, Chungcheongbuk-do 361-711, Korea

Tel: +82-43-269-6019, Fax: +82-43-273-325, E-mail: smpark@chungbuk.ac.kr

Financial support: None.

Conflicts of interest: None.

Author contributions: Gi Hyun Kim and Seon Mee Park planned this study and wrote the paper; Hyo Deok Lee, Min Kim, Kyeongmin Kim, Yusook Jeong, Yong Joo Hong, Eun Seok Kang and Jae-Woon Choi performed the research; Joung-Ho Han analyzed the data.

ORCID: Seon Mee Park, http://orcid.org/0000-0002-5835-2741. 


\section{Introduction}

The symptoms of gallstones occur in a wide range, from no symptoms to severe abdominal pain. ${ }^{1}$ In addition to abdominal pain, gallbladder stimulation by gallstones can give rise to gastrointestinal symptoms such as indigestion, nausea, vomiting and food intolerance. ${ }^{2}$ In almost all patients with gallstones, biliary pain disappears after cholecystectomy. ${ }^{3-5}$ However, the rates of relief from nonspecific gastrointestinal symptoms tend to be low and heterogeneous in patients who undergo an elective cholecystectomy.

In contrast to patients with gallstones, most patients with gallbladder polyps are asymptomatic. However, a minority of patients report abdominal symptoms that are similar to those induced by small gallstones that are rarely detached from the mucosa. ${ }^{6}$

When cholecystectomy is recommended, many patients wondered about the relief of their symptoms and about any occurrence of new symptoms after removing the gallbladder. However, many studies have reported that the changes in abdominal symptoms after laparoscopic cholecystectomy (LC) may differ according to the selection of patients and their preoperative conditions. Variable factors were reported as predictors of poor outcomes in many different studies. The presence of gastrointestinal diseases including irritable bowel syndrome ${ }^{7}$ or bloating, ${ }^{8}$ rather than the original gallbladder disease, was also related to the persistence of symptoms after cholecystectomy. Patients with psychiatric medication, ${ }^{8}$ psychological problems, ${ }^{8}$ personality disorders ${ }^{9}$ and neuroticism ${ }^{10}$ showed poor outcomes. Patients with poor outcomes after cholecystectomy also showed decreased compliance, which complicates the effectiveness of treatment. Therefore, a method for predicting outcomes and factors related to poor outcomes is necessary in the clinical setting.

This study was designed to evaluate the outcomes of nonspecific gastrointestinal symptoms after LC and to identify the factors attributable to poor outcomes. We compared symptom scores in patients with gallstones, who had typical biliary pain, and in those with gallbladder polyps, who were typically symptom free or experienced only mild abdominal discomfort. We evaluated the effects of psychological factors on the symptom outcome by scoring mental sanity in all patients with the Symptom Checklist-90 (SCL-90) questionnaire prior to surgery.

\section{Materials and Methods}

\section{Subjects}

Among 134 patients who received LC at Chungbuk National University Hospital between October 2009 and May 2010. A total 65 patients which include 32 patients with gallstones, 31 patients with gallbladder polyps and 2 patients with both gallstones and gallbladder polyps were enrolled in this study. Two patients with gallstones and gallbladder polyps were included in the gallstone group. All patients experienced relief from abdominal pain at the time of surgery and underwent elective LC. Patients were excluded for the following reasons: 19 who were younger than 15 or older than 65 years of age, 31 with acute cholecystitis, 8 with stenosis or gallstones in the biliary tract, 6 with presence of cancer and 5 with severe comorbidities. Written informed consent was obtained from all subjects prior to LC.

\section{Dyspeptic or Colonic Symptoms at Pre- and Post-operation}

Interviews were conducted 1 day before surgery to assess medical history and abdominal symptoms. Abdominal symptoms of indigestion, nausea, vomiting, food intolerance, heartburn, acid regurgitation, loss of appetite, diarrhea and constipation were recorded as 0, 1 and 2 for "none," "weak" and "severe," respectively. Additionally, simple psychological diagnoses and examinations using the Symptom Checklist-90-Revised (SCL-90-R) questionnaire were recorded for all patients to evaluate overall mental health prior to surgery. The SCL-90-R records 0 to 4 points for each item by self-evaluation, with measurements consisting of 90 items. Overall, the global severity index, positive symptom distress index, and positive symptom total were measured. The SCL-90-R used internet scoring (testcenter.co.kr) operated by Test Center Inc. (Seoul, South Korea). Total scores for abdominal symptoms were recorded at 3 and 6 months after surgery through phone interviews and compared with preoperative scores. Patients were determined to have an overall improvement when they remained in an asymptomatic state or showed a decrease in total symptom scores.

\section{Pathologic Evaluation of Cholecystectomized Gallbladder}

All gallbladder specimens were sent for histopathology. The presence of chronic cholecystitis, gallbladder wall thickening and 
adenomatous hyperplasia were analyzed as pathological data. Chronic cholecystitis was diagnosed when the mucosa was infiltrated with mononuclear inflammatory cells and the epithelium showed atrophic, hyperplastic or metaplastic changes. A gallbladder wall $\geq 3 \mathrm{~mm}$ in thickness was defined as thickened. Adenomatous hyperplasia was defined by the presence of hyperplasia of the gallbladder wall, limited to the epithelial elements of the mucosa without the involvement of the muscular layer. ${ }^{11}$

\section{Statistical Methods}

Discrete variables, including symptoms and clinical features, were evaluated using the Chi-square test, while the $t$ test or paired $t$ test was used to assess changes in symptom scores before and after surgery. SCL-90-R results were analyzed using the Mann-Whitney $U$ test to assess the relationship between patients' symptoms before and after surgery. $P$-values less than 0.05 were considered to be statistically significant. All analyses were performed using the SPSS software (ver. 12; SPSS Inc., Chicago, USA).

\section{Results}

\section{Characteristics of Patients With Gallstones or Gallbladder Polyps}

Among the 34 patients with gallstones or 31 patients with gallbladder polyps, no differences were noted for gender, ages, history of abdominal surgery, or history of pancreatitis or hepatitis. Two patients with gallstones were currently using a psychiatric medication. Twelve patients were diagnosed with gastritis by an endoscopic exam less than 2 months before surgery. All but 3 patients with gallbladder polyps had chronic cholecystitis. Gallbladder wall thickening more than $3 \mathrm{~mm}$ was detected in $67.6 \%$ and $54.8 \%$ of patients with gallstones and gallbladder polyps, respectively. Adenomatous hyperplasia was detected in seven patients with gallstones and in one patient with gallbladder polyp. Prior to surgery, all patients' blood and liver function tests were in normal ranges (Table 1).

\section{Patients' Dyspeptic or Colonic Symptoms Before and After Elective Laparoscopic Cholecystectomy}

One or more abnormalities in abdominal symptoms were recorded in $44(67.7 \%)$ patients, including indigestion (50.8\%), nausea $(26.2 \%)$, heartburn (23\%), loss of appetite $(21.5 \%)$, food intolerance $(20 \%)$, constipation $(18.5 \%)$, acid regurgitation (9.2\%), diarrhea (7.7\%) and vomiting (4.6\%) (Table 2). Abdominal symptom score (range, $0-18$ ) before surgery was 1.92 . This score decreased to 1.11 and 0.88 , at 3 months and 6 months after surgery, respectively $(P=0.006$ and $P<0.001$ by a paired $t$ test). The number of patients who showed improved symptom scores was $31(47.7 \%)$ and $36(55.4 \%)$ after 3 and 6 months,

Table 1. Clinical Characteristics of 65 Patients With Gallbladder Diseases

\begin{tabular}{lccc}
\hline \multicolumn{1}{c}{ Parameters } & Gallstones $(\mathrm{n}=34)$ & Gallbladder polyps $(\mathrm{n}=31)$ & $P$-value \\
\hline Male:Female & $16: 18$ & $19: 12$ & NS \\
Age (mean \pm SD, yr) & $45.0 \pm 7.0$ & $46.0 \pm 6.0$ & NS \\
Past medical history (n [\%]) & & & \\
Abdominal surgery & $11(32.4)$ & $11(35.5)$ & NS \\
Pancreatitis or hepatitis & $5(14.7)$ & $2(6.5)$ & NS \\
Psychiatric drugs & $2(5.9)$ & $0(0.0)$ & NS \\
Gastritis & $6(17.6)$ & $6(19.4)$ & NS \\
Laboratory findings (mean \pm SD) & & & NS \\
AST (IU/L) & $22.5 \pm 4.5$ & $23.6 \pm 6.6$ & 0.030 \\
ALT (IU/L) & $32.6 \pm 18.5$ & $154.5 \pm 66.8$ & NS \\
Alkaline phosphatase (IU/L) & $176.2 \pm 62.9$ & $6,319 \pm 1,811$ & NS \\
WBC (/mm $\left.{ }^{3}\right)$ & $6,650 \pm 1,388$ & & \\
Histopathological findings (n [\%]) & & $28(90.3)$ & NS \\
Chronic cholecystitis & $34(100.0)$ & $17(54.8)$ & NS \\
GB wall thicking $(\geq 3$ mm) & $23(67.6)$ & $1(3.2)$ & 0.033 \\
Adenomatous hyperplasia & $7(20.6)$ & &
\end{tabular}

AST, aspartate transaminase; ALT, alanine transaminase; WBC, white blood cell; GB, gallbladder; NS, not significant. 
respectively. Among abdominal symptoms, indigestion, food intolerance, heartburn and constipation were improved after 3 and 6 months. However, nausea, vomiting, loss of appetite and acid regurgitation did not differ before and after surgery. Diarrhea, which was aggravated 3 months after surgery, was restored to the pre-surgical level at 6 months after surgery (Table 2).

\section{Relation Between Dyspeptic or Colonic Symptoms and Psychological Symptom Scores}

A simple psychiatric test before surgery showed great differences between symptom positive and negative group. Specifically, patients with abdominal symptoms had significantly higher val-

Table 2. Frequencies of Dyspeptic or Colonic Symptoms Before and After Laparoscopic Cholecystectomy

\begin{tabular}{|c|c|c|c|c|c|}
\hline Symptoms & Before & 3 months after surgery & 6 months after surgery & $P_{1 \text {-value }}$ & $P_{2 \text {-value }}$ \\
\hline Absence & $21(32.3)$ & $42(64.6)$ & $45(69.2)$ & - & - \\
\hline Overall symptom scores (mean $\pm \mathrm{SD}$ ) & $1.92 \pm 2.11$ & $1.11 \pm 1.78$ & $0.88 \pm 1.68$ & $0.006^{\mathrm{a}}$ & $<0.001^{\mathrm{a}}$ \\
\hline Indigestion & & & & 0.035 & 0.013 \\
\hline None & $32(49.2)$ & $46(70.8)$ & $50(76.9)$ & & \\
\hline Weak & $20(30.8)$ & $15(23.1)$ & $11(16.9)$ & & \\
\hline Severe & $13(20.0)$ & $4(6.1)$ & $4(6.2)$ & & \\
\hline Nausea & & & & NS & NS \\
\hline None & $48(73.8)$ & $57(87.7)$ & $57(87.7)$ & & \\
\hline Weak & $15(23.1)$ & $8(12.3)$ & $8(12.3)$ & & \\
\hline Severe & $2(3.1)$ & $0(0.0)$ & $0(0.0)$ & & \\
\hline Vomiting & & & & NS & NS \\
\hline None & $62(95.4)$ & $65(100.0)$ & $62(95.4)$ & & \\
\hline Weak & $3(4.6)$ & $0(0.0)$ & $3(4.6)$ & & \\
\hline Severe & $0(0.0)$ & $0(0.0)$ & $0(0.0)$ & & \\
\hline Food intolerance & & & & 0.006 & $<0.001$ \\
\hline None & $52(80)$ & $51(78.5)$ & $55(84.6)$ & & \\
\hline Weak & $7(10.8)$ & $14(21.5)$ & $8(12.3)$ & & \\
\hline Severe & $6(9.2)$ & $0(0.0)$ & $2(3.1)$ & & \\
\hline Heart burn & & & & 0.014 & 0.024 \\
\hline None & $50(76.9)$ & $55(84.6)$ & $57(87.7)$ & & \\
\hline Weak & $14(21.5)$ & $10(15.4)$ & $8(12.3)$ & & \\
\hline Severe & $1(1.5)$ & $0(0.0)$ & $0(0.0)$ & & \\
\hline Acid regurgitation & & & & NS & NS \\
\hline None & $59(90.8)$ & $61(93.8)$ & $62(95.4)$ & & \\
\hline Weak & $5(7.7)$ & $4(6.2)$ & $3(4.6)$ & & \\
\hline Severe & $1(1.5)$ & $0(0.0)$ & $0(0.0)$ & & \\
\hline Loss of appetite & & & & NS & NS \\
\hline None & $51(78.5)$ & $54(83.1)$ & $61(93.8)$ & & \\
\hline Weak & $13(20.0)$ & $9(13.8)$ & $4(6.2)$ & & \\
\hline Severe & $1(1.5)$ & $2(3.1)$ & $0(0.0)$ & & \\
\hline Diarrhea & & & & 0.050 & NS \\
\hline None & $60(92.3)$ & $51(80.0)$ & $59(86.2)$ & & \\
\hline Weak & $5(7.7)$ & $10(15.4)$ & $9(13.8)$ & & \\
\hline Severe & $0(0.0)$ & $3(4.6)$ & $0(0.0)$ & & \\
\hline Constipation & & & & $<0.001$ & $<0.001$ \\
\hline None & $53(81.5)$ & $56(86.2)$ & $57(87.7)$ & & \\
\hline Weak & $9(13.8)$ & $9(13.8)$ & $8(12.3)$ & & \\
\hline Severe & $3(4.6)$ & $0(0)$ & $0(0.0)$ & & \\
\hline
\end{tabular}

${ }^{\mathrm{a}}$ Paired $t$ test.

$P_{1}, P$-value of before 3 months vs. post 3 months; $P_{2}, P$-value of before 6 months vs. post 6 months; NS, not significant.

Data are presented as $\mathrm{n}(\%)$. 
Table 3. Comparison of Psychological Symptom Scores Between Symptomatic and Asymptomatic Patients Before Laparoscopic Cholecystectomy

\begin{tabular}{lccc}
\hline Symptom Checklist-90-Revised & Symptomatic patients $(\mathrm{n}=44)$ & Asymptomatic patients $(\mathrm{n}=21)$ & $P$-value \\
\hline Somatization & $46.9 \pm 6.9$ & $39.0 \pm 2.8$ & $<0.001$ \\
Obsessive-compulsion & $41.3 \pm 8.0$ & $36.1 \pm 4.7$ & $<0.001$ \\
Interpersonal sensitivity & $42.4 \pm 8.7$ & $36.9 \pm 3.3$ & $<0.001$ \\
Depression & $42.4 \pm 7.9$ & $36.4 \pm 4.5$ & $<0.001$ \\
Anxiety & $44.2 \pm 7.0$ & $40.1 \pm 4.6$ & 0.015 \\
Hostility & $43.6 \pm 6.9$ & $40.5 \pm 2.2$ & 0.060 \\
Phobic anxiety & $45.2 \pm 6.8$ & $42.1 \pm 1.6$ & 0.148 \\
Paranoid ideation & $41.8 \pm 6.0$ & $39.4 \pm 3.5$ & 0.040 \\
Psychoticism & $42.6 \pm 7.2$ & $39.8 \pm 2.9$ & 0.063 \\
Global severity index & $42.3 \pm 7.6$ & $36.4 \pm 3.6$ & $<0.001$ \\
Positive symptom distress & $46.9 \pm 8.7$ & $39.9 \pm 12.3$ & 0.020 \\
Positive symptom total & $39.9 \pm 8.2$ & $31.4 \pm 6.2$ & $<0.001$ \\
\hline
\end{tabular}

Data are presented as mean $\pm \mathrm{SD}$ of $\mathrm{T}$ score.

Table 4. Clinical and Epidemiological Characteristics of Patients With Worsened Abdominal Symptoms at 6 Months After Laparoscopic Cholecystectomy

\begin{tabular}{|c|c|c|c|c|}
\hline Parameters & $\mathrm{n}$ & Improved patients $(\mathrm{n}=53)$ & Unimproved patients $(\mathrm{n}=12)$ & $P$-value \\
\hline Male & 35 & $29(82.9)$ & $6(17.1)$ & NS \\
\hline Age $($ mean $\pm \mathrm{SD}$, yr $)$ & 65 & $45.8 \pm 6.7$ & $45.0 \pm 6.4$ & NS \\
\hline Types of gallbladder diseases & & & & NS \\
\hline Gallbladder stones & 34 & $26(76.5)$ & $8(23.5)$ & \\
\hline Gallbladder polyps & 31 & $27(87.1)$ & $4(12.9)$ & \\
\hline Education status & & & & NS \\
\hline Less than college & 43 & $36(83.7)$ & $7(16.3)$ & \\
\hline University graduates & 22 & $17(77.3)$ & $5(22.7)$ & \\
\hline Socioeconomic status & & & & NS \\
\hline Less than intermediate & 14 & $11(78.6)$ & $3(21.4)$ & \\
\hline Medium or more & 51 & $42(82.4)$ & $9(17.6)$ & \\
\hline Marriage & & & & NS \\
\hline Unmarried & 10 & $7(70.0)$ & $3(30.0)$ & \\
\hline Married & 55 & $46(83.6)$ & $9(16.4)$ & \\
\hline \multicolumn{5}{|l|}{ Past medical history } \\
\hline Abdominal surgery & 22 & $18(81.8)$ & $4(18.2)$ & NS \\
\hline Pancreatitis or hepatitis & 7 & $7(100.0)$ & $0(0.0)$ & NS \\
\hline Psychiatric treatment & 2 & $1(50.0)$ & $1(50.0)$ & NS \\
\hline Gastritis & 12 & $6(50.0)$ & $6(50.0)$ & 0.007 \\
\hline \multicolumn{5}{|l|}{ Histopathologic findings } \\
\hline Chronic cholecystitis & 62 & $51(82.3)$ & $11(17.7)$ & NS \\
\hline GB wall thicking ( $\geq 3 \mathrm{~mm}$ ) & 40 & $33(82.5)$ & $7(17.5)$ & NS \\
\hline Adenomatous hyperplasia & 8 & $5(62.5)$ & $3(37.5)$ & NS \\
\hline Patients' satisfaction & & & & $<0.001$ \\
\hline Very satisfied & 38 & $38(100.0)$ & $0(0.0)$ & \\
\hline Satisfied & 16 & $12(75.0)$ & $4(25.0)$ & \\
\hline Neutral & 10 & $3(30.0)$ & $7(70.0)$ & \\
\hline Dissatisfied & 1 & $0(0.0)$ & $1(100.0)$ & \\
\hline
\end{tabular}

GB, gallbladder; NS, not significant.

Data are presented as n (\%). 
A

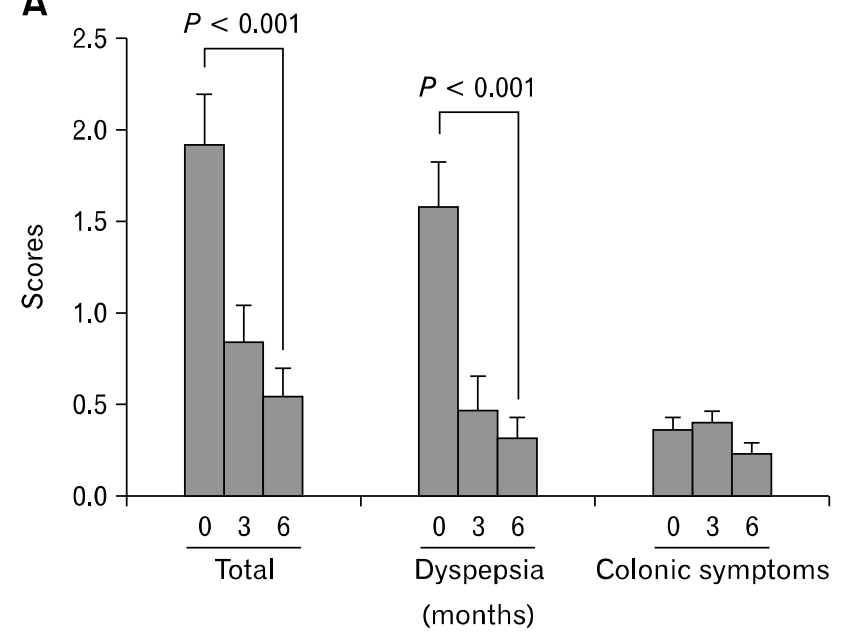

B

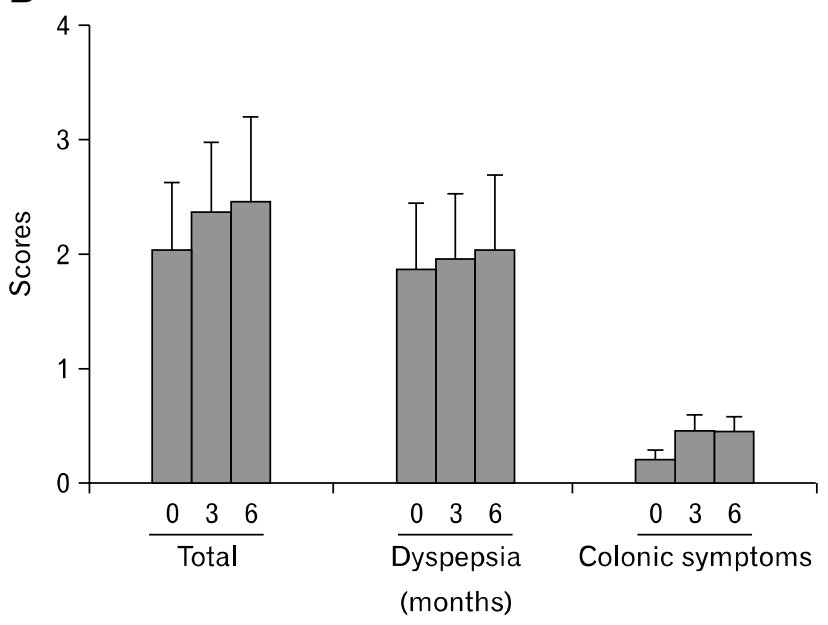

Figure. Changes of symptom scores, total, dyspeptic or colonic symptoms, before surgery and at 3 and 6 months after surgery in patients without gastritis (A) and in those with gastritis (B). Dyspeptic symptoms include indigestion, nausea, vomiting, food intolerance, heart burn, acid regurgitation and loss of appetite. Colonic symptoms represent diarrhea and constipation.

ues than patients without symptoms regarding somatization, obsessive-compulsion, interpersonal sensitivity, depression, anxiety, and paranoid ideation. Overall group measurements were also higher regarding global severity index, positive symptom distress index, and positive symptom total. However, no differences were noted between the groups for measures of hostility, phobic anxiety, and psychoticism (Table 3).

\section{Clinical Features of Patients With Continuing or Exacerbated Symptoms After Surgery}

At 6 months after cholecystectomy, 12 of 65 (18.5\%) patients had either no improvement or an increased total symptom score compared to before surgery. No difference was noted between improved and unimproved patients in regard to gallstones or gallbladder polyps, gender, age, educational levels, economic conditions, marital status, gallbladder pathology or history of abdominal surgery. However, the patients having gastritis showed poor outcomes ( $P=0.007$, Table 4$)$. The patients without gastritis showed total symptom scores to be decreased at 3 months and 6 months after LC compared to those at the time of enrollment, especially regarding the dyspeptic symptoms. However, the patients with gastritis did not show improvements of dyspeptic or colonic symptoms (Figure). Patients' surgical satisfaction differed depending on the degree of symptom improvement after surgery ( $P<0.001$, Table 4 ). Only $33.3 \%$ of patients with unimproved abdominal symptoms were satisfied with their surgery, while $94.3 \%$ of patients with symptom improvement were
Table 5. Changes in Psychological Symptom Scores According to Gastrointestinal Symptom Improvement at 6 Months After Laparoscopic Cholecystectomy

\begin{tabular}{lccc}
\hline $\begin{array}{c}\text { Symptom } \\
\text { Checklist-90-Revised }\end{array}$ & $\begin{array}{c}\text { Improved } \\
\text { patients } \\
(\mathrm{n}=53)\end{array}$ & $\begin{array}{c}\text { Unimproved } \\
\text { patients } \\
(\mathrm{n}=12)\end{array}$ & $P$-value \\
\hline Somatization & $45.3 \pm 7.5$ & $44.6 \pm 5.2$ & $\mathrm{NS}$ \\
Obsessive-compulsion & $40.2 \pm 7.4$ & $39.3 \pm 9.3$ & $\mathrm{NS}$ \\
Interpersonal sensitivity & $41.6 \pm 8.2$ & $41.8 \pm 8.9$ & $\mathrm{NS}$ \\
Depression & $42.2 \pm 8.0$ & $41.1 \pm 8.2$ & $\mathrm{NS}$ \\
Anxiety & $43.2 \pm 6.9$ & $43.0 \pm 6.4$ & $\mathrm{NS}$ \\
Hostility & $42.9 \pm 5.5$ & $44.2 \pm 10.2$ & $\mathrm{NS}$ \\
Phobic anxiety & $44.8 \pm 6.7$ & $44.3 \pm 4.6$ & $\mathrm{NS}$ \\
Paranoid ideation & $41.4 \pm 5.5$ & $41.8 \pm 7.7$ & $\mathrm{NS}$ \\
Psychoticism & $42.3 \pm 7.2$ & $42.1 \pm 4.9$ & $\mathrm{NS}$ \\
Global severity index & $41.1 \pm 7.4$ & $40.9 \pm 8.2$ & $\mathrm{NS}$ \\
Positive symptom distress & $45.2 \pm 10.5$ & $43.2 \pm 12.9$ & $\mathrm{NS}$ \\
Positive symptom total & $38.3 \pm 9.2$ & $37.6 \pm 7.3$ & $\mathrm{NS}$ \\
\hline
\end{tabular}

NS, not significant.

Data are presented as mean $\pm \mathrm{SD}$ of $\mathrm{T}$ score.

satisfied. No significant difference was detected in SCL-90-R examination values with respect to the patients' outcomes (Table 5).

\section{Discussion}

This study demonstrated that elective LC improves nonspecific gastrointestinal symptoms; the number of patients having abdominal symptoms decreased from $44(67.7 \%)$ before surgery, 
to $23(35.4 \%)$ and then $20(30.8 \%)$, respectively, at 3 and 6 months after surgery. Scores for symptoms before and after surgery showed the same trend, with the average score of 1.92 before surgery decreasing to 1.11 and 0.88 at 3 and 6 months, respectively. Symptoms were improved in patients with gallstones or gallbladder polyps, regardless of the type of disease.

Previous studies revealed that abdominal symptoms continued in $43-57 \%$ even after elective LC in patients with gallstones. ${ }^{2,8,12,13}$ In contrast, the present study showed a lower rate of continuous symptoms than those of previous studies. The reasons may be that all patients except three had chronic cholecystitis and severe pathologic changes are usually related with a high rate of symptom relief. ${ }^{14}$ Similar to previous reports, ${ }^{3,8,15,16}$ our findings showed that indigestion, food intolerance, heartburn, and constipation were improved after surgery. However, nausea, vomiting, and acid regurgitation did not differ before and after surgery. The incidence of diarrhea increased temporarily at 3 months after surgery and then recovered by 6 months. Currently, no studies have reported any variations in symptoms before and after surgery for patients with gallbladder polyps. This study confirmed that more than half of the patients with gallbladder polyps also reported dyspeptic or colonic symptoms before surgery and showed improvements after surgery which is a similar feature presented in patients with gallstones.

Nevertheless, cholecystectomy was not effective in easing the symptoms for every patient, because no symptom improvement or a worsening of symptoms occurred in 12 of 65 (18.5\%) patients at 6 months after surgery. Abdominal symptoms may have resulted from diseases in organs other than the gallbladder because many patients with no improvement in symptoms were diagnosed with gastritis before surgery. Sosada et al. ${ }^{17}$ reported that many symptoms had originated from gastrointestinal tracts and not from the gallbladder. In the present study, twelve patients were diagnosed with gastritis by endoscopy and these patients showed poor outcomes. Other studies revealed that the presence of irritable bowel syndrome ${ }^{7}$ or bloating ${ }^{8}$ was also related with persistent symptoms after cholecystectomy.

At present, no obvious correlation can be drawn from the literature regarding psychiatric problems and worsening symptoms after surgery. Previous studies suggested that patients with continuous pain after surgery had increased psychological vulnerability. ${ }^{14,18,19}$ However, other study reported that psychiatric factors had no special relationship with symptom variation after surgery. ${ }^{11}$ The current study considered that psychiatric conditions before a surgery did not greatly affect variation in symp- toms after surgery, although they showed significant differences when compared with the scores for patients with no symptoms.

Cholecystectomy has been recommended under limited conditions. ${ }^{20,21}$ Although controversy remains whether cholecystectomy should be performed in gallstone patients with dyspeptic or colonic symptoms, many reports show that atypical symptoms are improved after cholecystectomy in gallstone patients, suggesting the need to offer cholecystectomy to these patients. ${ }^{22}$ Considering that these studies found chronic cholecystitis in $95 \%$ of cases after cholecystectomy, atypical abdominal symptoms are likely to occur as a result of chronic inflammation. However, it is very difficult to identify patients with gallstones or gallbladder polyps for treatment when they report atypical abdominal symptoms, because symptom variation is very subjective. Although the causes of dyspeptic or colonic symptoms in patients with gallbladder polyps or gallstones have not been clarified, the present view is that surgery should be performed when other medical treatments have been tried and after the existence of gastritis is ruled out. In the present study, patient's satisfaction on surgery differed depending on the degree of symptom improvement after surgery.

Approximately two-thirds of patients who underwent gallbladder surgery showed improvement in their abdominal symptoms irrespective of gallstones or gallbladder polyps. Many patients with no improvement in their symptoms had been diagnosed with gastritis before surgery and their satisfaction with surgery was low. Detailed history-taking regarding gastritis before surgery can be helpful in predicting patients' outcome after LC.

\section{References}

1. Lorusso D, Porcelli P, Pezzolla F, et al. Persistent dyspepsia after laparoscopic cholecystectomy. The influence of psychological factors. Scand J Gastroenterol 2003;38:653-658.

2. Finan KR, Leeth RR, Whitley BM, Klapow JC, Hawn MT. Improvement in gastrointestinal symptoms and quality of life after cholecystectomy. Am J Surg 2006;192:196-202.

3. Berger MY, Olde Hartman TC, Bohnen AM. Abdominal symptoms: do they disappear after cholecystectomy? Surg Endosc 2003; 17:1723-1728.

4. Bitzer EM, Lorenz C, Nickel S, Dörning H, Trojan A. Assessing patient-reported outcomes of cholecystectomy in short-stay surgery. Surg Endosc 2008;22:2712-2719.

5. Yun SN, Kim SH, Park SJ, Jang JY, Park YH. Clinical outcome after laparoscopic cholecystectomy. J Korean Surg Soc 1999;57(suppl): 1023-1030.

6. Kwon W, Jang JY, Lee SE, Hwang DW, Kim SW. Clinicopathologic features of polypoid lesions of the gallbladder and risk factors of gallbladder cancer. J Korean Med Sci 2009;24:481-487.

7. Kirk G, Kennedy R, McKie L, Diamond T, Clements B. Preoper- 
ative symptoms of irritable bowel syndrome predict poor outcome after laparoscopic cholecystectomy. Surg Endosc 2011;25:3379-3384.

8. Luman W, Adams WH, Nixon SN, et al. Incidence of persistent symptoms after laparoscopic cholecystectomy: a prospective study. Gut 1996;39:863-866.

9. Svebak S, Søndenaa K, Hausken T, Søreide O, Hammar A, Berstad A. The significance of personality in pain from gallbladder stones. Scand J Gastroenterol 2000;35:759-764.

10. Jess $\mathrm{P}$, Jess $\mathrm{T}$, Beck H, Bech P. Neuroticism in relation to recovery and persisting pain after laparoscopic cholecystectomy. Scand J Gastroenterol 1998;33:550-553.

11. Stokes MC, Burnette R, Ballard B, Ross C, Beech DJ. Adenomatous hyperplasia of the gallbladder. J Natl Med Assoc 2007;99:959-961.

12. Wilson RG, Macintyre IM. Symptomatic outcome after laparoscopic cholecystectomy. Br J Surg 1993;80:439-441.

13. Ure BM, Troid $\mathrm{lH}$, Spangenberger W, et al. Long-term results after laparoscopic cholecystectomy. Br J Surg 1995;82:267-270.

14. McMahon AJ, Ross S, Baxter JN, et al. Symptomatic outcome 1 year after laparoscopic and minilaparotomy cholecystectomy: a randomised trial. Br J Surg 1995;82:1378-1382.

15. Lublin M, Crawford DL, Hiatt JR, Phillips EH. Symptoms before and after laparoscopic cholecystectomy for gallstones. Am Surg
2004;70:863-866.

16. Vignolo MC, Savassi-Rocha PR, Coelho LG, et al. Gastric emptying before and after cholecystectomy in patients with cholecystolithiasis. Hepatogastroenterology 2008;55:850-854.

17. Sosada K, Zurawinski W, Piecuch J, Stepien T, Makarska J. Gastroduodenoscopy: a routine examination of 2,800 patients before laparoscopic cholecystectomy. Surg Enndosc 2005;19:1103-1108.

18. Borly L, AndersonI B, Bardram L, et al. Preoperative prediction model of outcome after cholecystectomy for symptomatic gallstones. Scand J Gastroenterol 1999;34:1144-1152.

19. Jørgensen T. Abdominal symptoms and gallstone disease. An epidemiological investigation. Hepatology 1989;9:856-860.

20. Schmidt M, Dumot JA, Søreide O, Søndenaa K. Diagnosis and management of gallbladder calculus disease. Scand J Gastroenterol 2012;47:1257-1265.

21. Bang S. [Natural course and treatment strategy of gallbladder polyp.] Korean J Gastroenterol 2009;53:336-340. [Korean]

22. Mentes BB, Akin M, Irkörücü $\mathrm{O}$, et al. Gastrointestinal quality of life in patients with symptomatic or asymptomatic gallstones before and after laparoscopic cholecystectomy. Surg Endosc 2001;15:12671272. 